\title{
dsDNA-coated quantum dots
}

\author{
Protocol For: \\ dsDNA-coated quantum dots
}

Ming Xing, Huaibin Shen, Wei Zhao, Yanfei Liu, Yingda Du, Zhenxiang Yu, and Xia Chen College of Life Science, Jilin University, Changchun City, Jilin Province, China

BioTechniques Protocol Guide January 2017; doi 10.2144/000114498

See full protocol online: https://benchtalk.biotechniques.com/users/8521-biotechniques-editors/posts/11623-protocol-for-dsdna-coatedquantum-dots

Protocol Summary: We developed a versatile QD label by modifying dsDNA with biotin and thiol groups at opposite ends and attaching it to quantum dots via a metal-thiol bond. These dsDNA-coated QDs fluorescently label their targets through biotin-streptavidin binding and show excellent histological results when used to detect biotin-labeled chromosome probes. The dsDNA coating also circumvented the common problems of aggregation and steric hindrance that occur with other QD probes.

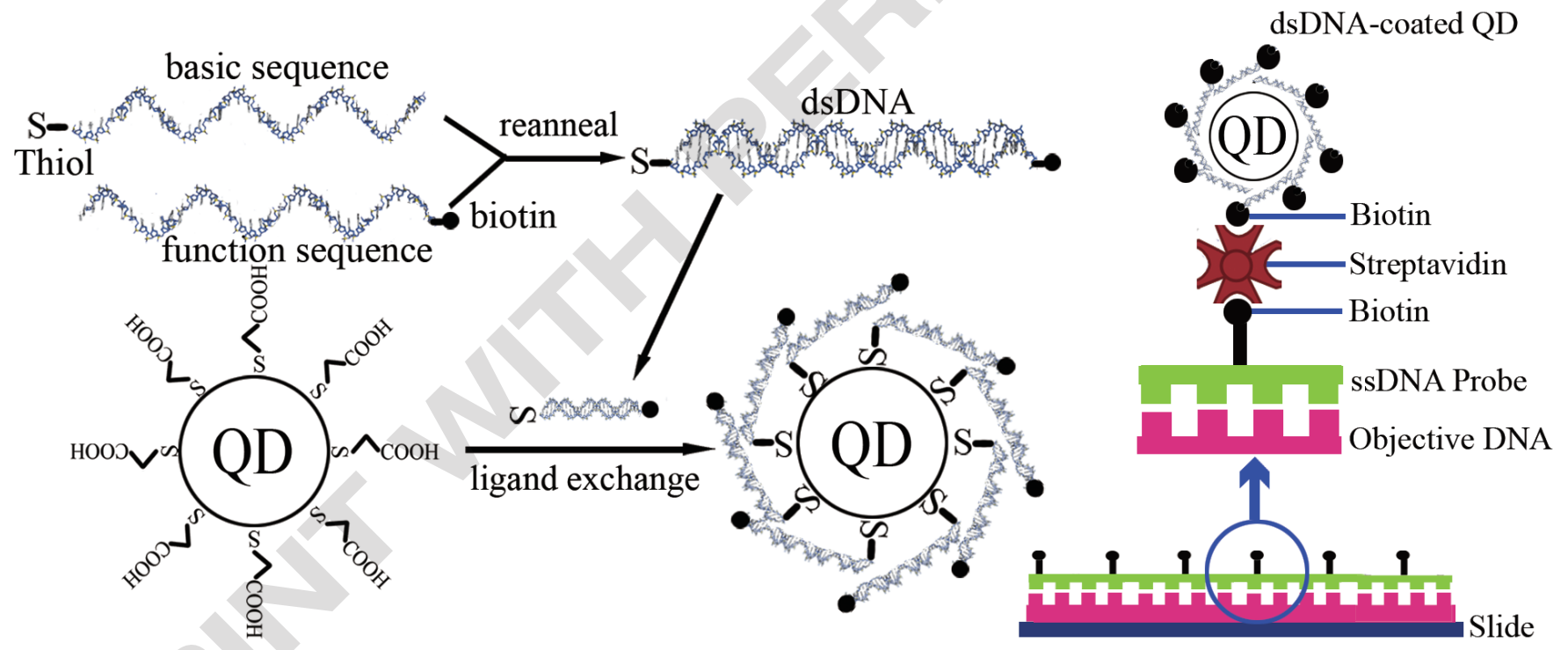

Protocol Schematic: Schematic presentation of the preparation of dsDNA-coated QD probes and the detection assay. (A) The thiolated sequence (base sequence) and its complementary biotinylated sequence (function sequence) anneal to form dsDNA containing a thiol group on one end and biotin on the other. (B) The thiol group on the dsDNA is exchanged with MAA carried on QDs via a metal-thiol bond. The dsDNA then wraps around the QDs to form a complete coat. (C) A sequence-specific ssDNA probe carrying a biotin group hybridizes with the target DNA sequence smeared on slides. Following hybridization, streptavidin is applied to the slides, allowing one streptavidin binding site to attach to the biotin carried on the probe and another binding site to anchor the biotin on the dsDNA-coated QDs. 